\title{
The importance of differentiating behavioural and psychological treatment effects from placebo in respiratory interventions
}

\section{To the Editor:}

We read with interest the piece by PatTinson and WANIGASEKERA [1], discussing the clinical implications of the recent trial reported by CURROw et al. [2], which demonstrated parity between sertraline and placebo for improving breathlessness intensity. In particular, Pattinson and Wanigasekera [1] noted that differential response expectations can potentially confound outcomes between otherwise balanced intervention versus placebo-controlled groups. Such expectations have clinical implications: the management of response expectations should be examined and potentially harnessed to improve treatment outcomes for patients with chronic breathlessness, as suggested by SimiLOWSKI and SERRESSE [3].

In his excellent paper, TuRner [4] reiterated that a placebo-controlled trial compares a particular component of a treatment (often primarily pharmacological) while attempting to keep all other therapeutically relevant components identical. Ideally, such an approach precludes bias and is the gold standard to develop evidence-based medical treatments. However, chronic breathlessness (amongst other complex long-term conditions) is increasingly recognised as dysfunction across multiple interconnected systems. As such it requires holistic treatment that, alongside physiological factors, addresses social, psychological, neurocognitive and behavioural aspects of disease. Therefore, it must be acknowledged that although placebo-controlled trials are designed to examine one specific treatment component, multiple components will impact patient outcomes, and these components may be present in both the treatment and placebo group.

This issue is particularly relevant in respiratory research, in which studies have consistently demonstrated very limited associations between objective physiological impairment and the subjective perceptions of breathlessness severity $[5,6]$. Thus, treatment benefits may impact patients through a diverse and complex interacting set of mechanisms (such as neurocognitive predispositions towards subjective symptom severity, as suggested by Ongaro and Kaptchuk [7]), which Pattinson and Wanigasekera [1] rightly note may not be balanced between placebo-controlled trial arms. Pertinently, we do not wish to devalue "traditional" randomised controlled trials. However, evidence from such trials should be considered alongside other types of research methods, such as "pragmatic" research that more closely reflects routine clinical care and "additively" evaluates the incorporation of a new treatment to patients' existing care, carefully monitoring all aspects of the new treatment (e.g. interaction with healthcare, subsequent self-management) in order to understand the likely mechanisms by which treatment benefits are conferred.

Many medical professionals think of a "placebo" as an inert pill but, as TURNer [4] noted, a comparison with "placebo" in a trial is merely a comparison of one group with another - and the "placebo pill" is merely a symbolic object involved in this process. This process definition is in line with modern accounts of clinical placebos focussed on context, meaning and embodiment. We also agree with the point made by Pattinson and WanigaseKera [1] that "placebo" is often used disparagingly to refer to treatments that do not work; such a position risks overlooking the benefits that are conferred independently to identified physiological improvements. This is particularly relevant for nonpharmacological treatments, such as

@ERSpublications

Well-designed nonpharmacological interventions can harness treatment responses seen in the placebo arm http://ow.ly/EgoM30o7k1v

Cite this article as: Ainsworth B, Hardman D, Thomas M. The importance of differentiating behavioural and psychological treatment effects from placebo in respiratory interventions. Eur Respir J 2019; 53: 1900156 [https://doi.org/10.1183/13993003.00156-2019]. 
breathing retraining exercises, mindfulness-based treatments or cognitive behavioural therapy, which are increasingly used as effective patient treatments in respiratory disease but demonstrate no measurable effect on physiological outcomes. By defining placebos as "inert" substances we are in danger of classifying such complementary treatments as ineffective, when in fact they may be cost-effective adjunct treatments that offer tangible patient benefits.

We welcome the call for "open-label" placebo research. However, a considered and cautious approach should be taken, insofar as such treatment may merely demonstrate the presence of broad psychological and behavioural treatment effects without determining which of these can be targeted (and therefore impacted by well-designed nonpharmacological interventions). Moreover, as CuRRow et al. [8] note (supported by modern contextual accounts of the placebo phenomenon [9]), although the results of small-scale open-label placebo trials may reflect genuine therapeutic benefit, they may also just be an artefact of the experimental situation, meaning it is unclear how such a treatment response can be harnessed.

These effects of open-label placebos are not caused by the "inert" pill itself, but by the construction and exploitation of a whole treatment process. Given that many existing nonpharmacological interventions can be conceived of as already established whole treatment processes (without the negative connotations of placebos) we advocate that the best way to therapeutically employ beneficial treatment responses may be to conduct careful research on these nonpharmacological interventions.

Ben Ainsworth ${ }^{1}$, Doug Hardman ${ }^{2}$ and Mike Thomas ${ }^{2}{ }^{2}$

${ }^{1}$ Dept of Psychology, Faculty of Humanities and Social Sciences, University of Bath, Bath, UK. ${ }^{2}$ Primary Care and Population Sciences, Faculty of Medicine, University of Southampton, Bath, UK.

Correspondence: Ben Ainsworth, Dept of Psychology, University of Bath, Claverton Down, Bath, BA2 7AJ, UK.

E-mail: b.ainsworth@bath.ac.uk

Received: Jan 222019 | Accepted after revision: Feb 232019

Conflict of interest: B Ainsworth has nothing to disclose. D. Hardman has nothing to disclose. M. Thomas has received speaker's honoraria for speaking at sponsored meetings or satellite symposia at conferences from the following companies marketing respiratory and allergy products: GSK and Novartis; has received honoraria for attending advisory panels with: Boehringer Ingelhiem, GSK and Novartis; is a recent a member of the BTS SIGN asthma guideline steering group and the NICE asthma diagnosis and monitoring guideline development group.

\section{References}

1 Pattinson K, Wanigasekera V. Sertraline or placebo in chronic breathlessness? Lessons from placebo research. Eur Respir J 2019; 53: 1802225.

2 Currow DC, Ekström M, Louw S, et al. Sertraline in symptomatic chronic breathlessness: a double blind, randomised trial. Eur Respir J 2019; 53: 1801270.

3 Similowski T, Serresse L. Lessons from negative dyspnoea studies: arguments for the multidimensional evaluation of multidirectional therapeutic approaches. Eur Respir J 2019; 53: 1802471.

4 Turner A. "Placebos" and the logic of placebo comparison. Biology \& Philosophy 2012; 27: 419-432.

5 Teeter JG, Bleecker ER. Relationship between airway obstruction and respiratory symptoms in adult asthmatics. Chest 1998; 113: 272-277.

6 Janssens T, Verleden G, De Peuter S, et al. Inaccurate perception of asthma symptoms: a cognitive-affective framework and implications for asthma treatment. Clin Psychol Rev 2009; 29: 317-327.

7 Ongaro G, Kaptchuk TJ. Symptom perception, placebo effects, and the Bayesian brain. Pain 2019; 160: 1-4.

8 Currow DC, Agar M, Ekström M. Sertraline or placebo in chronic breathlessness? Lessons from placebo research. Eur Respir J 2019; 53: 1802316.

9 Miller FG. Reining in the placebo effect. Perspect Biol Med 2018; 61: 335-348. 